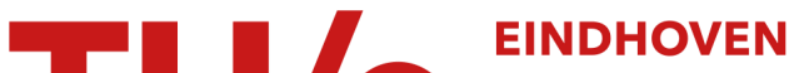 \\ UNIVERSITY OF \\ TECHNOLOGY
}

\section{The structuring of production control systems}

Citation for published version (APA):

Bertrand, J. W. M., \& Wijngaard, J. (1986). The structuring of production control systems. International Journal of Operations and Production Management, 6(2), 5-20. https://doi.org/10.1108/eb054756

DOI:

10.1108/eb054756

Document status and date:

Published: 01/01/1986

\section{Document Version:}

Publisher's PDF, also known as Version of Record (includes final page, issue and volume numbers)

\section{Please check the document version of this publication:}

-A submitted manuscript is the version of the article upon submission and before peer-review. There can be important differences between the submitted version and the official published version of record. People interested in the research are advised to contact the author for the final version of the publication, or visit the $\mathrm{DOI}$ to the publisher's website.

- The final author version and the galley proof are versions of the publication after peer review.

- The final published version features the final layout of the paper including the volume, issue and page numbers.

Link to publication

\section{General rights}

Copyright and moral rights for the publications made accessible in the public portal are retained by the authors and/or other copyright owners and it is a condition of accessing publications that users recognise and abide by the legal requirements associated with these rights.

- Users may download and print one copy of any publication from the public portal for the purpose of private study or research.

- You may not further distribute the material or use it for any profit-making activity or commercial gain

- You may freely distribute the URL identifying the publication in the public portal.

If the publication is distributed under the terms of Article 25fa of the Dutch Copyright Act, indicated by the "Taverne" license above, please follow below link for the End User Agreement:

www.tue.nl/taverne

Take down policy

If you believe that this document breaches copyright please contact us at:

openaccess@tue.nl

providing details and we will investigate your claim. 


\title{
The Structuring of Production Control Systems
}

\author{
by J. W. M. Bertrand and J. Wijngaard \\ Eindhoven University of Technology, The Netherlands
}

Received August 1985

Revised November 1985

This paper presents a qualitative methodology for designing hierarchically structured production control systems for complex production situations. The methodology is based on the assumption that complexity should be reduced by defining self-contained subsystems with clear and well-defined operational characteristics. Furthermore the interactions between the subsystems should be simple and restricted. We introduce the Production Unit ( $P U)$ as a basic control entity. From the perspective of goods flow control the PUs are black boxes having certain operational characteristics. The objective of goods flow control is to realise a certain delivery performance, taking into account the $P U$-operational constraints. The main elements in the goods flow control structure as developed here are Master Planning, Material Co-ordination, Workload Control and Work Order Release.

\section{Introduction}

Production control refers to the co-ordination of production and distribution activities in a manufacturing system to achieve a specific delivery reliability at minimum costs. In many customer oriented production situations the manufacturing activities have developed in such a way that manufacturing is specialised according to product-type and/or to manufacturing technology. The result is a production structure with a number of production units, where each unit takes care of a separate part of the production, and where the goods flow in and between these production units can be quite complex. In such a production system, each of the production units will have its own shortterm and long-term goals, whereas each product-type delivered to the market may require materials and capacity from a number of different production units.

In order to realise the required delivery performance in the market, co-ordination of the activities of the production units is therefore necessary. These co-ordination activities, however, should not conflict with reaching the production economics objectives for each of the production units. On the one hand, realising production economics objectives is in the interest of the system as a whole. On the other hand, however, the production units should show high flexibility with respect to reacting to changing market conditions, demand forecasts, and actual demand. Lack of flexibility may lead to high and unbalanced stocks, poor delivery performance, and possibly loss of market position. This conflict between short-term interests of operation units 
and goods flow control is well-known in the literature. A structural (hierarchical) approach is needed to resolve this conflict[1].

In the past decade a number of studies have been published on the design of hierarchical production control systems. Many of these studies reported on the principles underlying particular design projects in practice[e.g., 2, 3]. Other research used mathematical analysis to investigate specific types of aggregation and decomposition[e.g., 4-6], or used systematic computer simulation for this purpose[e.g., 7, 8]. In this paper we stress the general problem of how to structure the complete production control process. We study the subject from the point of view that the control sub-problems at any level should be defined such that the controllability of the problem is guaranteed and the actual control performance can be measured and therefore can be monitored. This approach has been used in a previous research project on production control in a production unit [9] and is now applied to a more complex production problem including goods flow control. The concepts used in this approach are partly based on certain concepts from Manufacturing Resources Planning (MRP-II).

This conceptual framework for production control deals with the problems of shortterm and long-term inflexibilities in the production units $[10,11]$. Specifically, the concept of a Master Production Schedule (MPS) has been introduced as a device to reconcile the conflicts between market needs and production possibilities (see, e.g.[12]).

The MRP-II concepts were a substantial step forward in the design of goods flow control systems. However, a real quantitative basis for the operational design of such systems is still lacking, as can be concluded from the many difficulties encountered when MRP-II is being used as a basis for design in practice. This paper aims at filling a part of this practicality gap. It introduces some basic concepts for designing production control śystems which achieve high flexibility while still enabling the production units to realise their production economics.

For this purpose, we first introduce definitions of basic concepts for describing a production system, such as items, materials, capacity and operations, then we introduce the concept of Production Unit which is used as a homogeneous logistic entity. The co-ordination of the production units is referred to as Goods Flow Control (GFC). Next we consider how to structure GFC and the relationship of GFC to Production Unit Control (PUC).

\section{Basic Concepts}

We assume that for any production control problem in practice a system boundary can be determined. The system boundary defines what "part of the production world" is considered and what part is beyond our scope. The system boundary should be established operationally by specifying the inflows and outflows from the environment into the system considered and vice versa. This system boundary concept is illustrated in Figure 1.

We assume the manufacturing processes to be given. Thus for each finished product (end item) the following is known:

- the end product structure, which is the way in which the product is composed of materials, parts or components, and subassemblies;

- the capacity types needed; 
- the manufacturing steps which are needed for each of the components, subassemblies and final assemblies in the product; and

- the amount of capacity required for each manufacturing step.

Figure 1. The Boundaries of the Production System

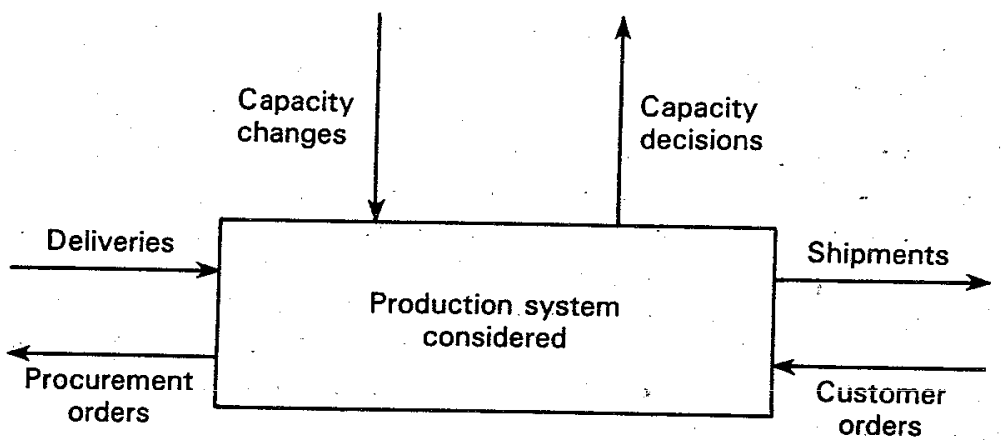

With this general specification of the system we can roughly define the production control problem to be that given certain consistent objectives regarding customer delivery performance and manufacturing costs, how should we:

(1) accept customer orders;

(2) place procurement orders;

(3) vary the capacity;

(4) allocate available capacity to manufacturing steps?

Depending on the system boundary chosen, the complexity of the problem can vary substantially. We restrict our research to production systems with many complex enditems, where interactions and relationships between products and their timing stem from the following factors:

- the products use shared capacity resources, with restricted availability;

- the products use shared types of materials and subassemblies;

- information on market demand is limited;

- work orders are released with batch sizes which may be larger than the immediate required amount;

- short term capacity variations are possible to a limited extent and with a certain leadtime.

\section{Materials, Resources}

We assume that the manufacturing process for an end-item can be defined as a related set of transformations. Each transformation may require materials and/or resources. As materials we define other items which are absorbed in a finite discrete amount 
during the transformation step. As a resource we define objects which are not used up during the transformation step, but which are in use (machines, space, etc.).

\begin{abstract}
Operations
A manufacturing process is a network of manufacturing steps. For the purpose of production control the manufacturing steps are aggregated into operations. The specification of operations should be related to the scope of the production control problem at hand. The operations generally do not follow straightforwardly from the description of the manufacturing steps, but must be based on the aspect of the system that is addressed by production control. Now as production control addresses the timing of the allocation of resources and materials, a natural criterion for the grouping of manufacturing steps into operations is their relative independence in time. Thus if there is little freedom in relative timing within a group of manufacturing steps, it would be natural to consider this group as one operation, which requires the resources and materials of all the manufacturing steps in the group. From the production control point of view, an operation is a biack box with specific properties, and which is not subject to internal manipulation.
\end{abstract}

\title{
Production Units and Goods Flow Control
}

In order to simplify the total production control it is necessary to distinguish production units (PU). A PU is a specific set of capacities performing a specific set of operations using a specific set of materials. The total control is decomposed to PU Control (PUC) and Goods Flow Control (GFC). The control variable which constitutes the interface between PUC and GFC is the release of new work orders to the PU. The PU has to realise the work orders according to certain norms. GFC has to co-ordinate the release of work orders to the various PUs, to each other and to customer orders and to provide the required materials.

An ideal PU is self-contained from a manufacturing point of view. Also from a production control point of view the PU is self-contained, but it is generally constrained with respect to the amount and timing of its production. These constraints constitute the operational relationships of a production unit. The constraints are basically generated by its limited capacity and by the operation processing times required for the manufacturing of the items. However, additional constraints can be generated by the way in which a PU organises its production process in order to realise specific objectives regarding product quality and production efficiency. For instance, if set-up times are an important part of the operation processing time, then the PU may want to work with specific batch sizes. Moreover, if machine set-up times are sequence dependent, the PU may want to maintain a certain working stock of work-in-process at that machine in order to be able to create an efficient production sequence.

The creation of a PU requires a relatively stable environment for that unit with respect to the availability of resources and the demand for product items produced by that unit. This stability is required because the PU will operate in a relatively independent way, and therefore it will need a number of environmental invariabilities to base its internal structure on. 
From the perspective of Goods Flow Control, the PUs are black boxes, which have specific operational characteristics, and which can only be influenced under certain conditions via specific inputs. These conditions reflect the agreements regarding the environmental conditions. For instance, an agreement could be that GFC can release work orders to a PU, on the condition that the work load of that PU never exceeds a specific limit. On that condition, the PU may promise average delivery times of started work orders according to specific pre-set norms. A very different agreement might be that GFC could release any work order to the production unit, and that the PU promises to deliver the orders according to variable due dates, specified at the time of release, which takes into account the actual workload at that time. Many more examples can be given of possible sets of agreements regarding performance and environmental conditions.

It will be clear that generating stable environmental conditions for a PU will be quite easy if the environment of the production system itself is rather stable. In fact, the "difference" between the actual stability of the system's environment and the stability implied in the agreed environmental conditions of the PUs has to be accounted for by GFC. For instance, if the agreed conditions per PU imply more stability than the system's environment shows, then the GFC system should be designed so that it can absorb the difference. Goods Flow Control could then hold and use buffer stocks of finished goods or components to allow for the PU to adapt to the changes in the environment in a smooth way. On the other hand, if the agreements with the PU imply much flexibility, then GFC can just pass the variations in the system's environment to the PU.

Each possible transformation that can be realised by a PU can be defined on the production network of operations. Ideally, a PU should be defined such that the subnetworks per PU can be considered from the GFC viewpoint as single production phases. Then, for GFC, the production processes for end-items can be expressed in terms of this set of production phases, and a set of relationships between these phases. In fact these sets constitute an aggregate production structure, which allows the GFC to use a rather aggregate production model showing much less detail. GFC does not deal with operations, but with production phases. Thus GFC controls the behaviour of the stock levels of the items at specific points in the operations network of the products. These specific points are the manufacturing states in between the PUs. We refer to these manufacturing states as controlled stock items of the production process.

The GFC problem therefore can be defined as the control of the levels of the controlled stock items, by means of the release of work orders for production phases, within the constraints set by the PU agreements, in order to realise a specific delivery. performance at minimum costs.

Bertrand[13] and Bertrand and Wijngaard[14] give more details about production units, the interface of PUC and GFC and the location of controlled stock-points.

\section{Goods Flow Control Structure}

Considering the complexity of Goods Flow Control, it will be clear that designing a general optimal control is impossible. We propose to decompose GFC into the following four parts: 
(1) Master Planning

(2) Material Co-ordination

(3) Workload Control

(4) Work Order Release.

Figure 2 gives an outline of the relationship between these levels of control. The Master Planning level forms the connection with the higher levels of control in the production organisation, where the various systems of the organisation (logistics, quality, finance, manpower, etc.) are integrated into a production objective (Management Control[15]). This production objective (the Master Plan) is formulated in terms of production level plans for the PUs and a delivery schedule for the Master Production Schedule (MPS) items. Material Co-ordination translates the MPS into priorities for purchasing orders and production work orders per controlled stock item, taking into account the actual production progress. Finally, the production level plans per PU are combined with the work order priorities to determine which work orders are to be released to the shop floor if the actual workload allows such a release. The operational constraints of the PU are accounted for in this phase. From this description it will be clear that Workload Control and Work Order Release are decision functions located at the interface between Goods Flow Control and Production Unit Control.

Depending on the specific production situation this global control structure can be detailed in many different ways. Two factors are particularly crucial in this respect:

- the co-ordination of sales and manufacturing;

- the interference of capacities and products.

These two elements will be discussed next.

Figure 2. Sketch of Goods Flow Control Structure

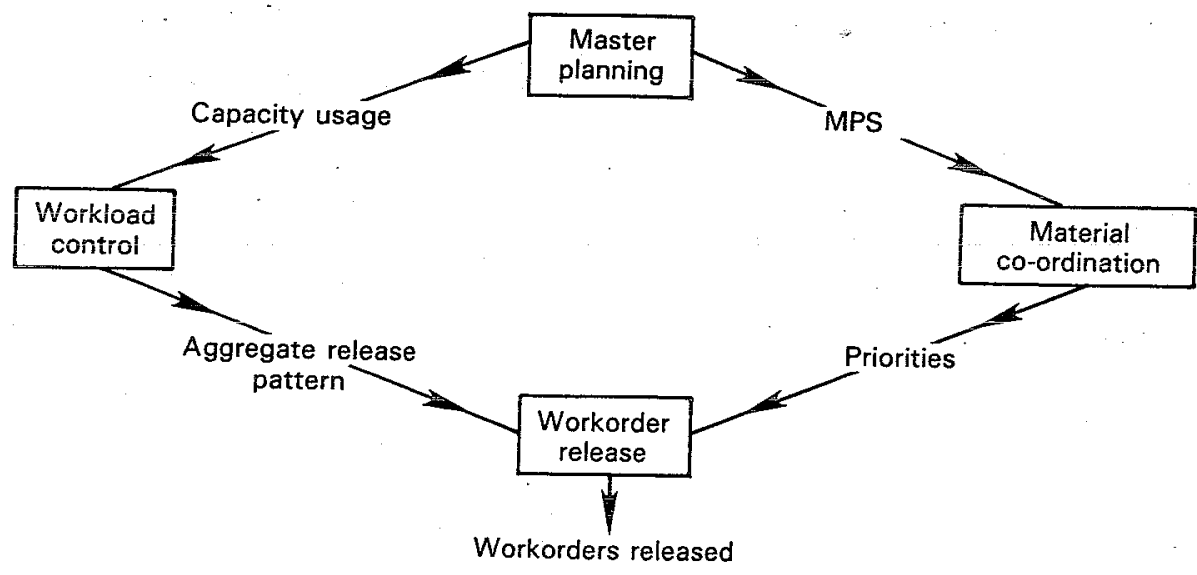




\section{Co-ordination of Sales and Manufacturing}

Within each production system one has to recognise the influence of the sales and manufacturing functions. Sales generates demand and accepts commitments with respect to customer deliveries. Manufacturing has to realise the required deliveries. Co-ordination between these activities is required. There have to be aggregate agreements with respect to service performance, stability and reliability of required delivery patterns, total accepted demand, etc. This we call structural co-ordination, which affects aggregates and averages.

\section{Structural and Operational Co-ordination}

If there is only structural co-ordination sales determines required delivery patterns, taking into account the aggregate agreements, but neglecting the actual state of manufacturing. In such a situation the required deliveries can be interpreted as autonomous demand. In many cases, however, improvements can be expected from also taking into account the actual state of manufacturing in the co-ordination process. This implies operational co-ordination. In the MRP-II framework this type of co-ordination is incorporated in the concept MPS[12]. However, in that framework the MPS serves two purposes. It is not only a realistic (potential) delivery plan based on the structural and operational co-ordination of sales and manufacturing, but it is also the basis for co-ordinating the various production units in the system by netting and offsetting. In current MRP literature thee two functions of the MPS are not well distinguished. In this paper we will formalise this distinction and interpret the MPS as a (potential) delivery plan based on state information with respect to both sales and manufacturing.

\section{Sales Flexibility}

Including state information with respect to manufacturing in the MPS makes sense only if sales can use the information. This is the case if there exists a certain flexibility at the sales side. Such sales flexibility could be for instance the possibility of influencing customer order due dates, the possibility of starting short term sales promotions, etc. Another source of sales flexibility exists if sales controls to some extent the inventories in the distribution stages. However, if sales flexibility is very limited it can be beneficial to have an MPS including information on the state of manufacturing. For instance if, due to some manufacturing problem, shortages are inevitable, it makes it possible for sales to distribute the shortages over the various product types or customers.

In situations where sales flexibility is very small the MPS ean be interpreted as "demand", that is, as an objective for manufacturing. In cases where there is much short term sales flexibility the MPS also represents the state of manufacturing and can be interpreted as an objective for sales as well.

\section{Form of the MPS}

An MPS is a sequence of vectors or a matrix (quantities/period of the MPS items). It will be clear that such a matrix is a rather poor representation of the combined state of the manufacturing and sales process. A better state representation would be 
a set of trajectories. For manufacturing these trajectories would pertain to the set of realisable delivery patterns; for sales they would pertain to the set of acceptable delivery patterns, where the term acceptable refers to the possibility of realising certain sales patterns. The objective of both sales and manufacturing would then be to realise a non-void intersection of these two sets of trajectories.

However, we should keep in mind that these state variables can only be realistically described in stochastic terms, as the real future deliveries and sales not only depend on the current state and the future control decisions, but also on a number of uncontrolled stochastic variables. Therefore we accept the convention of using one single delivery pattern as state representation of both manufacturing and sales. This pattern may (informally) be interpreted as a more or less arbitrary pattern in the abovementioned intersection of sets of realisable and acceptable delivery patterns. From this discussion it will be clear that the relationship between this MPS and the real state of the system cannot be formalised. However, in situations where the flexibility of manufacturing and sales and the procedures to co-ordinate both are quite stable, we may expect that the participants in this co-ordination process will generate implicitly stable and consistent models of this relationship.

Generally the MPS is established periodically and is intended to be valid during the following period. The discussion above makes clear that each new MPS may differ from the old one. This is because of all kinds of uncontrolled stochastic variables, with respect to sales as well to manufacturing, which have occurred during the period, and which are accounted for in the new MPS. By not adapting the MPS, a high sales or manufacturing flexibility would be presupposed and this flexibility would be used in a very rigid and probably non-optimal way.

\section{Interference of Capacities and Products}

To illustrate the relationship between capacities and products in Goods Flow Control we consider a production system with independent demand (no sales flexibility). The difficulty of having an MPS instead of responding to demand has been discussed in the previous section. In the next section both difficulties are combined and complete control structures are described.

In situations with independent demand the objective of GFC is to realise a certain service performance. This has to be done by controlling work order releases to PUs and by adjusting capacities and capacity usage of PUs. The decision freedom for GFC to manipulate these variables and to vary the inventories in the controlled stock points have to be "budgeted" by higher levels of control. This also includes the restrictions on the variables imposed by the batching and sequencing constraints.

In controlling the goods flow we face disturbances and fluctuations with respect to:

- procurement leadtimes

- production leadtimes

- capacities

- yield

- demand

- registration of inventory and work in process. 
In general it is important to put effort into reducing these fluctuations and disturbances or into making them more predictable. The current drive in industry for realising short leadtime, high quality (zero-defect) and just-in-time production therefore should be highly valued. However, in many situations the possibilities are limited, sometimes because of technical constraints, sometimes because of economic constraints. On top of that, the capacity flexibility may be too small to cope with the remaining variability, be it predictable or unpredictable. For many situations inventory buffers are necessary to absorb the state variations due to the gap between flexibility and variability. Using inventory buffers in the right way can be an effective and efficient way to absorb short term variability.

\section{Inventory as Stored Capacity.}

A weak point of using inventory buffers to absorb production and demand fluctuations is that inventories can only be realised as quantities of specific items, while most types of fluctuations are directly or indirectly related to capacity availability. Fluctuations in production leadtimes, for instance, are partly due to disturbances in capacity availability. But fluctuations in demand for a certain product also have a capacity dimension. To some extent, inventories of other products can be used to absorb these fluctuations. In short, inventory of specific products has the property that it also can be used as stored capacity.

We will clarify this point with a simple example. Consider a make-to-stock situation with one production stage and two products which have identical production and demand characteristics. Compare a state with inventories $\left\{I_{1}, I_{2}\right\}$ with a state with inventories $\left\{I_{1}+\mathrm{x}, \mathrm{I}_{2}-\mathrm{x}\right\}$.

In the short term these states are different with respect to the risk of stockout. On a somewhat longer view, however, the states are equivalent in this respect. The term at which the states are equivalent corresponds to the term at which for both products production has taken place. At that term the effectiveness of the buffer for absorbing capacity and demand fluctuations depends only on the total inventory. Also, fluctuations in demand for product 1 or product 2 are equivalent at that term. One can cope to the same extent with both types of fluctuations irrespective of the detailed state of the inventory.

\section{Two Control Levels}

The above example shows that in the somewhat longer term the inventory of products with regular demand can be considered as being just stored capacity. This term is roughly equal to the production cycle time, that is, the term at which all products with regular demand have been produced at least once. In many situations the production cycle time is much shorter than the term at which capacities can be changed. That makes it possible to distinguish two levels of control. Capacity decisions can be decomposed from detailed inventory control and work order release. In many cases, i.e., where constant work order throughput times are a prerequisite for adequate control, capacity usage is determined by capacity[9]. In other situations it is possible in principle to vary capacity usage without varying the capacity. In such situations work orders are directly coupled to customer orders and internal work order throughput times may be related to customer order due dates. However, in such situations, if the 
PUs operate at a high utilisation rate, such independent variations of capacity usage have a long lasting impact on mean work order throughput times. For that reason, in these cases it makes sense to integrate capacity decisions and capacity usage decisions and to decompose this from detailed inventory control.

This kind of decomposition has been investigated and discussed[16-18]. At the first level, only capacities and capacity aggregates are used as variables. Demand, production and inventory are all aggregated to capacity. At the second level, results with respect to production levels are used as budgets. Next, the production level has to be allocated to the various products. A good objective for this second level of control is to keep the expected run-out times of the individual products equal as far as possible (taking into account, of course, the batching and sequencing restrictions). At this level detailed short term information has to be used. Because of production and demand variations, and because of the operational constraints of the PUs it is not possible to realise completely equal run-out times. The remaining degree of imbalance in run-out times can be considered as the control performance of the second level and has to be taken into account at the first control level: at the first level extra inventory (slack) should be provided to allow for this imbalance.

The effectiveness of such a decomposition depends on the extent to which the performance of the second level decision process is independent of the decisions made at the first level. Independence is high in case of rigid capacities and high utilisation rates.

It should be noticed that slow moving products should be excluded from this hierarchical method of control. This is because slow mover inventory is not very effective as stored capacity. Including slow movers would increase the production time significantly. Thus the problem remains of how to deal with slow movers in this approach. A straightforward and very effective possibility is to give slow movers high priority at all levels of decision making and to adjust the capacity availability by reducing it for the capacity required for the slow movers (for details of this approach see[19]).

Decomposition can also be applied to multi-stage production situations[20], although in this case the relationship between the capacity aspect and the product aspect is more intricate. In this case, for each product-item of a PU the horizon for the second (detailed) level of Goods Flow Control has to be increased with the total production leadtime (the stacked item leadtime).

\section{The Product-oriented Approach}

An attractive alternative to the capacity-oriented heirarchical approach discussed above is the product-oriented approach. In this approach the capacity usage decisions are not integrated with the capacity adjustment decision. Capacity usage is integrated with capacity allocation. These decisions are decomposed along the products; all products are controlled separately. The interference with other products because of restricted capacities is modelled as a stationary extension of the production leadtime in the PU. This interference is revealed at the work order release level. In situations with a stable utilisation rate such a product-oriented approach works just as well as the hierarchical approach[18]. It is possible then to use queueing type analyses to estimate the delays due to the interference of products because of restricted capacities[21]. But if there is no stable utilisation rate the product-oriented approach may not be expected to work well. Decisions with respect to capacity changes have to be based on the effect of these 
changes at the lower level of control. The only effect which can be taken into account easily in this product-oriented approach is the influence on utilisation rate and via utilisation rate on interference delays. This only makes sense if each capacity change leads to a new stationary situation. However, if a PU works at a high utilisation rate, the transient times are long and it will take much time before a new situation is established. Thus if capacities are changed frequently it is necessary to control (aggregate) inventories and capacities simultaneously. The product-oriented approach does not then fit.

It has to be mentioned that in the hierarchical approach slow movers have to be controlled in a product-oriented manner. However, the capacity usage of slow movers is generally so small that there are hardly any delays due to interference with the restricted capacities. This implies that with the capacity-oriented hierarchical approach we have none of the problems that are typical for the product-oriented approach.

\section{The Integral Detailed Approach}

Up to this point we have discussed two ways to decompose the complete problem of controlling capacities, capacity usage and individual product inventories. Our premises have been that the complexity and the stochastic nature of the problem make a decomposition approach unavoidable. However, in simple and more deterministic cases the use of an integral approach may be realistic, possibly restricted to the main products. An integral model of the production control problem, including decision variables, state variables and goal variables, can then be built, and the solution of this model can be realistically implemented in practice. In that case it is generally necessary to use a kind of decomposition but this time the term decomposition refers to techniques to solve the integral model (i.e., decomposition techniques applied to large scale mathematical programming models). See Billington et al.[22] for an outline of such an approach. This type of decomposition (problem decomposition) should be distinguished carefully from the decomposition approach applied to decision making processes which we discuss in this paper.

\section{The Complete Control Structure}

The Goods Flow Control structure has to depend on the:

- flexibility of the system

- objectives

- characteristics of the environment.

The flexibility of the GFC system is determined by the operational relationships of the Production Units and by restrictions (budgets) with respect to inventories, makeor-buy decisions, capacity changes, etc. The objectives of the GFC system are norms and restrictions with respect to service performance. Characteristics of the environment refer to procurement characteristics, customer behaviour, yield (product and production quality) behaviour of capacities (as far as not controllable by GFC).

It has to be mentioned that the distinction between an objective and a restriction of the system is rather arbitrary. For instance an inventory budget could be interpreted as an objective. However, we choose not to do so. We consider timely delivery as the objective function of GFC and the possibility of varying inventories as one of the means for realising this. 
To guarantee an adequate control structure and to realise consistent restrictions and objectives it is necessary (at design level) to monitor and control flexibility, objectives and environmental characteristics. It is important to notice that flexibility is not given but is partly the result of the definition of production units and the design of Production Unit Control. At GFC level PUs are characterised by operational constraints and norms. Flexibility within the PUs provides the possibility of keeping the characteristics rather simple[23]; this contributes to GFC simplicity. The introduction of PUs with rather simple operational constraints and performance norms reduces the variety of GFC systems. However, the variety is still too high to allow for one uniform structure to be applied to all possible situatons in practice. Therefore, we have developed two closely related structures which cannot be used in all situations directly, but which can, in most situations at least, be used as a starting point in the design process. The difference between the structures is due to different PU characteristics. The first structure is intended for cases where the production levels of the PUs are difficult to change (e.g. complex job-shop production units). The second structure is intended for situations where the production level can be varied at the same term as the capacity allocation. In both structures, however, we distinguish four levels of control:

- Master Planning

- Workload Control

- Material Co-ordination

- Work Order Release.

Here we will only present the first structure. For a description of the second structure and a discussion of the relationship of the two structures we refer to[14].

From now on we restrict our attention to cases where, in the short term, the production level of the PUs is inflexible. To keep the performance of such PUs predictable, the mean throughput time and utilisation rate should be fixed[9]. This means that the available capacity determines the production level. The basic structure we propose for this kind of situation is given in Figure 3. The function of Master Planning is to control capacity variations (and hence variations in production level) and the aggregate MPS. The capacity aggregates determined by Master Planning serve as restrictions for Material Co-ordination. The function of Material Co-ordination is to disaggregate (allocate) the aggregate flows so as to get a good balance of the individual inventories. The relationship between Master Planning and Material Coordination is as previously described. The performance of Material Co-ordination in such a structure is not expressed in terms of customer order delivery performance, but is related to the balance of the individual final inventories (or backlogs). At Master Planning level an estimate of this performance should be available to allow realisation of aggregate inventories such that the delivery performance for each of the individual products is sufficient.

Two important factors influencing the performance of Material Co-ordination are batching restrictions and sequencing restrictions.

Workload Control and Work Order Release are functions at the interface of GFC and PUC. The output of Workload Control is the aggregate release pattern. Work Order Release concerns work orders of individual products. Co-ordination with PUC 
is necessary because of preferences within the PU. We will consider each of these functions now in more detail.

Figure 3. Goods Flow Control Structure in the Case of Inflexible Production Levels

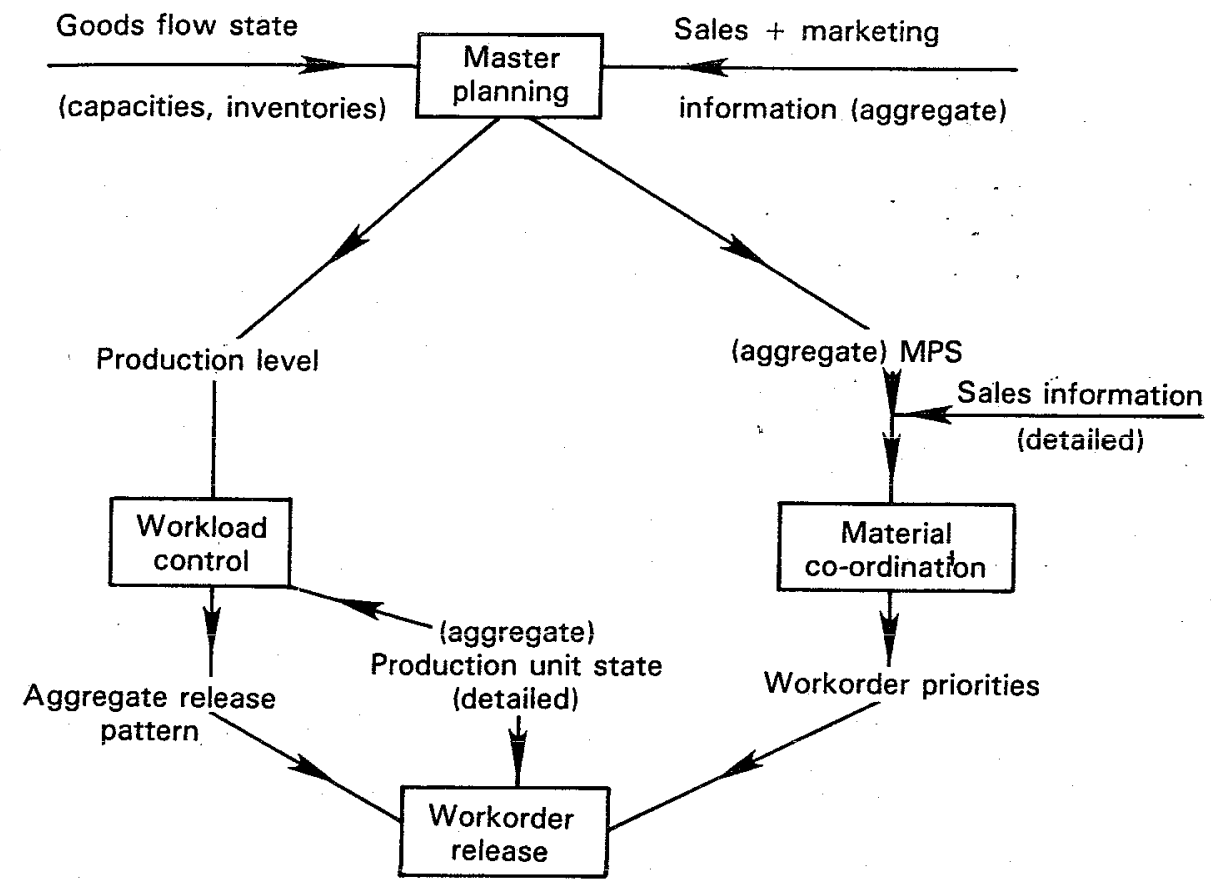

\section{Master Planning}

The aggregates to use at Master Planning level are capacity aggregates. This is easy as long as there is only one relevant capacity dimension and the products have almost identical characteristics. In such cases production level patterns and an aggregate MPS are sufficient to project future expected aggregate inventory patterns.

It may become more difficult if there are more relevant capacity dimensions. It is necessary then to estimate the capacity content of the inventories for all relevant capacity dimensions. This can be done by controlling the MPS in a more detailed way, or by using fixed ratios of capacity requirements on different capacity dimensions. This is equivalent to aggregating the bill of capacity. The possibilities of using such aggregate capacity bills in the Master Planning process have been investigated by Axsäter and Jönsson[8]. The stability of the MPS over the various products is important in using such aggregate bills.

Another difficulty with respect to aggregate Master Planning is due to the existence of slow movers or more general products with irregular production. As has been explained, such products should not be included in the aggregate decision variables. A possible solution is to aggregate only over regularly produced products and give 
the other products a high priority at all levels of decision making. The slow movers are then completely controlled by Material Co-ordination, and the control performance of Material Co-ordination with respect to these products is the delivery performance itself. The aggregate release pattern for fast movers has to be adjusted for the releases of work orders for slow movers.

It is difficult to construct suitable decision support models to support the Master Planning function. An important reason for this is that it is not easy to formalise sales flexibility. The MPS is the output of a co-ordination process of sales/marketing and manufacturing. Given the current definition of a Master Plan, the procedures of this co-ordination process can be formalised, but not its content. As long as sales and marketing flexibility cannot be formalised in an operational way it is only possible to support the production level determination of Master Planning by models in which the MPS is treated as independent demand. And even then the models may become highly complex because of the behaviour of the MPS as a function of time (stability, reliability) and because of the multi-stage, multi-capacity aspect of the production situation. However, one could try to use HMMS-rules, linear programmingbased or control theory type of decision rules, and to evaluate, by means of numerical analyses or simulation, how such methods work for certain typical situations. Evaluation has to be based on variation in production level and inventory variation[24]. The influence of the planning horizon could be one of the points of interest here (for reviews of models which can be used to support the Master Planning function see[25-27]).

\section{Material Co-ordination}

As already mentioned the function of Material Co-ordination is to balance the final inventories of the various products. The capacity usage determined by Master Planning serves as a budget. Slow movers, however, are excluded from this hierarchical approach and are controlled completely at Material Co-ordination level.

It is important to notice that at the Material Co-ordination level it is possible in general to use more actual and more detailed sales information than at the Master Planning level. Master Planning and Material Co-ordination may have different review periods and the sales information used by Material Co-ordination is detailed and updated compared to the aggregate sales information used by Master Planning.

Models to support Material Co-ordination are infinite capacity safety stock models [28] as far as products with irregular production are concerned. For products with regular demand one needs models to estimate the effect of certain release policies on the imbalance of final inventories. Constructing useful models of this last kind is fairly easy because the imbalance of the final inventories is rather insensitive to capacity variations and changes in predictability[29]. This even makes it possible to use small scale simulation models to get complete results for this control aspect.

\section{Workload Control}

In the type of situation considered here the throughput time is assumed to be determined at a higher level of control. That means that capacity, production level and workload norm are strictly coupled variables. Applying the workload norm means that the aggregate release pattern depends also on the way the PU is controlled. The aggregate release depends on the state of the PU. 


\section{Work Order Release}

Material Co-ordination determines detailed (dynamic) release priorities taking into account the (static) restrictions with respect to batching and sequencing. However, the preferences of the PU cannot be described completely by such static restrictions. Think for instance of the actual availability of specialised personnel. This means that in general the actual work order releases will be the result of a co-ordination process of Material Co-ordination and Production Unit Control. The actual state of the production unit will influence what is going to be released. This will affect the performance of Material Co-ordination (the balance), just as the sequencing and batching restrictions affect this performance. At the Master Planning level this effect has to be taken into account as well.

\section{Conclusions}

Production control is complex. Many decisions interfere with each other and the production control in total interferes with the control of other aspects in the organisation (quality, manpower,...). Structuring is necessary to reduce the complexity. The structure should be chosen so that the loss of potential flexibility is minimised.

The precise structure to be chosen should depend on the characteristics of the organisation. However, there are elements with respect to structure which have a much wider generality. The following elements have been discussed in this paper:

- the definition of basic elements as capacities, materials and operations as a first step in the design of the production control structure, instead of considering them as externally given items;

- the introduction of production units and the decomposition of the total production control to Goods Flow Control and Production Unit Control; and

- the relationship of sales and manufacturing and the interference of products and capacities as two main determining factors of the Goods Flow Control structure.

The generality of these elements makes it possible to develop a small, but relatively complete set of reference structures. For Goods Flow Control in a repetitive manufacturing situation (multi-stage, multi-product) we have discussed one such reference structure.

During recent decades the contribution of Operations Research (OR) to production control has been quite restricted. On the one hand $O R$ could contribute to solving specific instances of problems in practice. On the other hand OR could contribute to solving rather general but artificially simple theoretical problems. The existence of (more or less) standard production control structures makes it possible to exploit OR models and techniques much better. The existence of such standard structures also reduces the number of relevant OR models. Relevant OR models are models which fit some standard structure or which can be used to choose between different standard structures.

\section{References}

1. Meal, H. C., "Putting Production Decisions where they Belong", Harvard Business Review, Vol. 84, 1984, pp. 102-111.

2. Bitran, G. R. and Hax, A. C., "On the Design of Hierarchical Production Planning Systems", Decision Sciences, Vol. 8, 1977, pp. 28-55. 
3. Hax, A. C. and Meal, H. C., "Hierarchical Integration of Production Planning and Scheduling", in, M. A. Geisler (Ed.), Studies in Management Sciences, Vol. 1, Logistics, North-Holland-American Elsevier, Amsterdam, 1975.

4. Axsäter, S., "On the Design of the Aggregate Model in a Hierarchical Production Planning System", Engineering and Process Economics, Vol. 4, 1979, pp. 89-97.

5. Zipkin, P., "Exact Approximate Cost Functions for Product Aggregates", Management Science, Vol. 28, 1982, pp. 1002-12.

6. Wijngaard, J., "On Aggregation in Production Planning", Engineering Costs and Production Economics, Vol. 6, 1982, pp. 259-65.

7. Jönnson, H., "Simulation Studies in Hierarchical Systems in Production and Inventory Control", PhD Thesis, Department of Production Economics, Linköping University, 1983.

8. Axsäter, S. and Jönsson, H., "Aggregation and Disaggregation in Hierarchical Production Planning", European Journal of Operational Research, Vol. 17, 1984, pp. 338-50.

9. Bertrand, J. W. M. and Wortman, J. C., Production Control and Information Systems for Component Manufacturing Shops, Elsevier Scientific Publishing Co., Amsterdam, 1981.

10. New, C. C., Managing the Manufacture of Complex Products, Business Books, London, 1977.

11. Plossl, G. W. and Welch, W. E., The Role of Top Management in the Control of Inventory, Reston Publishing Co., Reston, VA, 1979.

12. Berry, W. L., Vollmann, T. E. and Whybark, D. C., Master Production Scheduling: Principles and Practice, APICS, 1979.

13. Bertrand, J. W. M., "A Hierarchical Approach to Structuring the Goods Flow Control in MultiPhase Production Systems", IFIP Working Group 07 Workshop, Copenhagen, August 29-31, 1984. (To be published in Proceedings.)

14. Bertrand, J. W. M. and Wijngaard, J., "The Structuring of Production Control Systems", Report ARW 03 THE BDK/ORS/84/10, Eindhoven University of Technology, 1984.

15. Anthony, R. N., Planning and Control Systems: A Framework for Analysis, Harvard University, Cambridge, Massachusetts, 1965.

16. van Beek, P., "An Application of Dynamic Programming and the HMMS Rule on Two-level Production Control", Zeitschrift für Operations Research, Vol. 21, 1977, pp. B133-B141.

17. Meal, H. C., "A Study of Multi-Stage Production Planning", in Hax, A. C. (Ed.), Studies in Operations Management, Chapter 9, North-Holland, Amsterdam, 1978.

18. Bemelmans, R. P. H. G. and Wijngaard, J., "A Capacity Oriented Approach of Production and Inventory Systems", Proceedings of the Second International Symposium on Inventories, 1982, pp. 595-607.

19. Bemelmans, R. P. H. G., "On the Capacity Aspect of Inventories", PhD Thesis, Department of Industrial Engineering, Eindhoven University of Technology, 1985.

20. Wijngaard, J., "Aggregation and Decomposition in Logistic Control of Multi-Stage, Multi-Product, Production/Inventory Systems", in J. P. Brans (Ed.), Operational Research '84, 1984, pp. 551-65.

21. Williams, T. M., "Special Products and Uncertainty in Production/Inventory Systems", European Journat of Operational Research, Vol. 15, 1984, pp. 46-54.

22. Billington, P. J., McClain, J. O. and Thomas, L. J., "Capacity Constrained MRP Systems", Management Science, Vol. 29, 1983, pp. 1126-41.

23. Simon, H. A., The Sciences of the Artificial, MIT Press, Cambridge, Massachusetts, 1981.

24. Bertrand, J. W. M., "Balancing Production Level Variations and Inventory Variations in Complex Production Systems", Report BDK/KBS/84/04, Eindhoven University of Technology, 1984.

25. Bemelmans, R. P. H. G., "A Single-Machine Multi-Product Planning Problem under Periodic Review", Report BDK/ORS/83/02, Eindhoven University of Technology, 1983.

26. Silver, E. A., "Aggregate Production Planning: State of the Art", Production and Inventory Management, 1st quarter, 1972, pp. 15-40.

27. Boskma, K., "Medium Term Planning of Production and Sales", PhD Thesis, University of Groningen (in Dutch), 1979.

28. Wortmann, J. C. and Wijngaard, J., "MRP and Inventories", Report BDK/ORS/84/08, Eindhoven University of Technology, 1984.

29. Wijngaard, J., "Capacities in Inventory Control", Report BDK/ORS/84/06, Eindhoven University of Technology, 1984. 